\title{
QUANTIFICAÇÃO DE COMPOSTOS BIOATIVOS EM FRUTOS DE UMBU (Spondias tuberosa Arr. Câm.) e CAJÁ (Spondias mombin L.) NATIVOS DE ALAGOAS
}

Everton Ferreira dos Santos; Rychardson Rocha de Araújo²; Eurico Eduardo Pinto de Lemos³; Laurício Endres³.

\author{
1Estudante de Pós-Graduação em Agronomia (PPGA), Centro de Ciências Agrárias (CECA), Universidade Federal de Alagoas (UFAL), \\ BR 104 Norte, Km 85, Rio Largo, AL. CEP 57.000-100. \\ ${ }^{2}$ Professor do Departamento de Engenharia Agrícola (DEAGRI/UFS), Av. Marechal Rondon, S/N, São Cristovão - SE. CEP \\ 49100-000. \\ ${ }^{3}$ Professor, Centro de Ciências Agrárias (CECA), Universidade Federal de Alagoas (UFAL), BR 104 Norte, Km 85, Rio Largo, AL. CEP \\ 57.000-100. \\ *Autor para correspondência: Everton Ferreira dos Santos, everton_federal@hotmail.com
}

RESUMO: o umbuzeiro e a cajazeira são fruteiras nativas de caráter extrativista, ocorrendo em áreas de restingas e caatingas do Estado de Alagoas, desempenhando importante papel como fonte de renda e complementação alimentar para as populações nos locais onde ocorrem. Seus frutos apresentam características de qualidade que os fazem ser muito apreciados tanto de forma in natura como processado. Contudo, em virtude da desordenada expansão agrícola e urbana estas espécies correm sérios riscos de serem extintas de Alagoas, necessitando de estudos que visem à caracterização de suas potencialidades. Dentro deste contexto, 0 trabalho teve como objetivo quantificar os teores de antocianinas, flavonoides, pectina total e pectina solúvel em frutos de S. tuberosa Arr. Câm. e S. mombin L. procedentes da vegetação nativa alagoana. Os teores de antocianinas e flavonoides foram determinados por meio da metodologia proposta por Francis (1982), onde se utilizou $1 \mathrm{~g}$ de polpa para cada espécie frutífera e solvente extrator solução de Etanol- $\mathrm{HCl}(85: 15 \%)$, sendo os resultados expressos em mg.100 $\mathrm{g}^{-1}$ de polpa. Com relação à quantificação de pectina total e solúvel foi utilizado o método do m-hidroxidifenil, utilizando $5 \mathrm{~g}$ de polpa de cada espécie frutífera e solvente extrator EtOH (95\%), e os resultados expressos em \%, segundo metodologia desenvolvida por McReady e MacComb (1952). As analises foram realizadas em quaduplicata, sendo obtidos os valores médios e desvio padrão para cada variável. 0 fruto de umbu apresentou maior teor de antocianinas totais com valor médio de 1,65 $\pm 0,47 \mathrm{mg} \cdot 100 \mathrm{~g}^{-1}$, sendo que o fruto de cajá apresentou maior conteúdo de flavonoide totais com teor médio de $9,50 \pm 0,71 \mathrm{mg}^{1} 100 \mathrm{~g}^{-1}$. Com relação aos teores de substâncias pécticas o fruto de umbu apresentou maior rendimento médio de pectina solúvel e pectina total, com valores de $28,64 \pm 2,47 \%$ e $26,26 \pm 4,35 \%$. Os frutos das duas espécies de fruteiras nativas de Alagoas analisados apresentam em sua composição química considerável conteúdo de compostos com propriedades biologicamente funcionais, constituindo-se em uma fonte potencial de antioxidantes naturais para a dieta humana. Eles também apresentaram bons teores de pectina total e solúvel, indicando seu potencial tanto para o consumo in natura quanto para o processamento agroindustrial.

PALAVRAS-CHAVE: Spondias, frutos nativos, compostos bioativos.

\section{QUANTIFICATION OF BIOACTIVE COMPOUNDS IN UMBU FRUITS (Spondias tuberosa Arr. Câm.) and CAJÁ (Spondias mombin L.) NATIVE TO ALAGOAS}

\begin{abstract}
: umbuzeiro and cajazeira are native fruit extractive species occurring in restingas and caatingas areas of the State of Alagoas, playing an important role as a source of income and food supplementation for the populations where they occur. Their fruits have characteristics of quality that make them very appreciated both in fresh and processed. However, due to the disorganized agricultural and urban expansion, these species are in serious danger of extinction in Alagoas, needing studies aimed at characterizing their potentialities. In this context, the objective of this study was to quantify anthocyanins, flavonoids, total pectin and soluble pectin in fruits of S. tuberosa Arr. Câm. and S. mombin L. from native Alagoan vegetation.
\end{abstract}


The levels of anthocyanins and flavonoids were determined using the methodology proposed by Francis (1982), where $1 \mathrm{~g}$ of pulp was used for each fruit species and solvent extracting Ethanol-HCl solution (85: $15 \%$ ), the results expressed in $\mathrm{mg} .100 \mathrm{~g}-1$ of pulp. Regarding the quantification of total and soluble pectin, the m-hydroxydiphenyl method was used, using $5 \mathrm{~g}$ of pulp of each fruit species and solvent extractor EtOH $(95 \%)$, and the results expressed in\%, according to methodology developed by McReady and MacComb (1952). The analyzes were performed in quad replicate, and the mean values and standard deviation for each variable were obtained. The fruit of umbu had a higher content of total anthocyanins with an average value of $1.65 \pm 0.47 \mathrm{mg} .100 \mathrm{~g}-1$, and the fruit of cajá had the highest total flavonoid content with a mean content of $9.50 \pm 0.71 \mathrm{mg} .100 \mathrm{~g}-1$. Regarding the pectic substances, the fruit of umbu showed a higher average yield of soluble pectin and total pectin, with values of $28.64 \pm 2.47 \%$ and $26.26 \pm 4.35 \%$. The fruits of the two native fruit species of Alagoas analyzed present in their chemical composition considerable content of compounds with biologically functional properties, constituting a potential source of natural antioxidants for the human diet. They also presented good levels of total and soluble pectin, indicating their potential for both in natura consumption and agroindustrial processing.

KEY WORDS: Spondias, native fruits, bioactive compounds.

\section{INTRODUÇÃO}

O Brasil é detentor de uma das maiores diversidades de espécies vegetais do planeta, que em virtude de sua extensão continental e localização geográfica, apresenta uma ampla faixa de variação de clima e solo, o que o torna privilegiado para a exploração de recursos genéticos de valor potencial atual ou futuro, tendo em vista o desenvolvimento de novas tecnologias. Porém, a grande riqueza vegetal existente vem sendo relegada pela pesquisa científica brasileira, especialmente para as espécies frutíferas nativas, que apresentam um enorme potencial de inserção no mercado como produto diferenciado quando forem devidamente investigadas (Teixeira et al., 2016; Silva et al., 2012).

O gênero Spondias, inserido na família botânica Anacardiaceae, reúne cerca de 18 espécies distribuídas nos neotrópicos, Ásia e Oceania, apresentando espécies nativas do Brasil. Dentre as espécies pertencentes a este gênero seis ocorrem no Nordeste, compreendendo espécies frutíferas tropicais de grande importância econômica devido ao valor comercial de seus frutos, e algumas também apresentam importância medicinal. As principais espécies que ocorrem no Nordeste brasileiro são: Spondias mombin L. (cajazeira), Spondias purpurea L. (sirigueleira), Spondias cytherea Sonn. (cajaraneira), Spondias tuberosa Arr. Câm. (umbuzeiro) e Spondias spp. (umbu-cajá e umbuguela) (Souza et al., 1999; Souza, 1998).
Dentre as espécies de ocorrência no Nordeste destaca-se S. tuberosa Arr. Câm. e S. mombin L., que são largamente exploradas por meio do extrativismo, constituindo-se em uma importante fonte de renda para as comunidades nos locais onde ocorrem. Também em pequena escala são cultivadas em pomares domésticos ou em pomares mal conduzidos, carecendo de estudos que tenham por objetivo sua domesticação e desenvolvimento de tecnologias que viabilizem a instalação de pomares comerciais (Menezes et al., 2017).

\section{Em Alagoas a cajazeira ocorre} naturalmente em áreas de restingas e caatingas, com frutificação concentrada entre os meses de janeiro a julho. 0 umbuzeiro é um fruto nativo do semiárido nordestino, considerado um sinônimo de resistência em meio às secas prolongadas, ocorrendo em Alagoas em áreas de caatinga, e sua frutificação também ocorre entre os meses de janeiro a julho (Santos, 2010).

No estudo da qualidade pós-colheita em frutos nativos, inúmeras características podem ser avaliadas, como $0 \mathrm{pH}$, acidez total, sólidos totais, teor de acido ascórbico, pectina total, pectina solúvel, antocianinas, flavonoides, atividade antioxidante, entre outros parâmetros. As informações sobre os parâmetros físico-químicos, nutricionais e funcionais constituem-se em ferramentas de qualidade importantes em frutos, pois contribui para o interesse da população pelo consumo dos mesmos, bem como para o desenvolvimento de novos produtos, 
oferecendo uma alternativa de atividade sustentável para as populações das regiões de ocorrência das espécies frutíferas nativas (Silva et al., 2015; Rocha et al., 2013; Canuto et al., 2010).

Os frutos de $S$. tuberosa Arr. Câm. e $S$. mombin L. são caracterizados por apresentar alto conteúdo de vitamina $\mathrm{C}$, excelentes características de sabor e aroma, aparência e qualidade nutritiva, e rendimento de polpa de aproximadamente $68 \%$, com grande potencial tanto para o consumo in natura como para o processamento agroindustrial, podendo ser consumidos na forma de sucos, doces, geleias e sorvetes. Contudo, verifica-se na literatura escassez de estudos relacionados à caracterização fitoquímica dos mesmos, sendo atributos de qualidade importantes para a inserção e aceitação destes frutos no mercado frutícola brasileiro (Lima et al., 2015).

Nos últimos anos tem-se verificado um crescente aumento no consumo de frutas pela população mundial, constituindo-se em uma importante tendência para as próximas décadas. Este fato se deve aos benefícios oferecidos pelas frutas ao organismo, principalmente devido à presença de constituintes químicos, com propriedades nutricionais e funcionais, que atuam na proteção do organismo contra os danos oxidativos causados pela exposição do mesmo aos radicais livres, e desta forma contribui para a redução ou prevenção de inúmeras doenças (Oliveira et al., 2016).

Os consumidores estão cada vez mais exigentes em relação à qualidade em frutos, o que tem se tornado um fator essencial para a conquista e ampliação de mercado. Alguns fatores que conferem boa qualidade aos frutos são alto teor de vitamina C, a presença de carotenoides, compostos fenólicos (antocianinas e flavonoides), e substancias pécticas. Estes compostos têm intensificado interesses, em função das importantes funções e ações que eles possuem para a saúde humana, principalmente por atuarem como antioxidantes e sequestrantes de radicais livres, capazes de ajudar a reduzir 0 risco de enfermidades como o câncer e doenças cardiovasculares (Pimentel et al., 2005).

Os frutos avaliados neste estudo apresentam um grande potencial econômico, uma vez que, apresentam-se bastante difundidos na alimentação cotidiana das populações nos locais onde ocorrem como também na produção de variados produtos. Diante do exposto, o trabalho teve como objetivo quantificar os teores de antocianinas, flavonoides, pectina total e pectina solúvel em frutos de $S$. tuberosa Arr. Câm. e S. mombin L. procedentes da vegetação nativa do Estado de Alagoas.

\section{MATERIAL E MÉTODOS}

\section{Local de execução e coleta do material vegetal}

0 estudo foi desenvolvido no Laboratório de Pós-colheita de Frutos do Centro de Ciências Agrárias da Universidade Federal de Alagoas (CECA/UFAL), localizado em Rio Largo - Alagoas. Os frutos foram coletados de plantas que ocorrem naturalmente em áreas de caatingas e restingas alagoanas. 0 clima da região onde os frutos foram coletados, segundo Köppen é classificado como tropical chuvoso, com verão seco e precipitação média anual de 1.150,2 mm, sendo os meses mais secos de novembro a dezembro e o mais chuvoso julho a agosto (SEMARH, 2015). Foram coletados \pm $800 \mathrm{~g}$ de frutos para cada espécie, em seguida foram acondicionados em caixa com isolamento térmico com gelo e transportados para o Laboratório de Póscolheita de Frutos - CECA/UFAL. Em laboratório os frutos foram lavados em água corrente e sanitizados em solução de hipoclorito de sódio $(5 \mathrm{~mL}$ de cloro: $1 \mathrm{~L}$ de água), por um período de 30 minutos, em seguida foi realizado o enxague para remover os resíduos de cloro. Em sequencia os frutos foram desintegrados em multiprocessador, obtendo-se $\pm 350 \mathrm{~g}$ de polpa para cada espécie. As polpas dos frutos foram mantidas a $-10^{\circ} \mathrm{C}$ até o momento das análises

\section{Determinação quantitativa dos teores de antocianinas e flavonoides totais por espectrofotometria.}

As antocianinas totais e os flavonoides totais foram determinados segundo a metodologia desenvolvida por Francis (1982). Onde se pesou 1 $\mathrm{g}$ de polpa do fruto em um Becker envolto de papel alumínio, em seguida, adicionou-se $30 \mathrm{~mL}$ da solução extratora etanol $(95 \%) / \mathrm{HCl}(1,5 \mathrm{~N})$ na proporção 85:15, previamente preparada. As amostras foram homogeneizadas em um homogeneizador de tecidos tipo "Turrax" por 2 minutos na velocidade "5". Logo após, o conteúdo foi transferido para um balão volumétrico 
de $50 \mathrm{~mL}$ (sem filtrar) ao abrigo da luz, aferido com a solução extratora, homogeneizado e armazenado em frasco âmbar, o qual ficou em repouso por uma noite na geladeira. No dia seguinte, o material foi filtrado em um kitassato de $100 \mathrm{~mL}$, acoplado a uma bomba de vácuo protegido da luz. As leituras foram realizadas em quaduplicata, em espectrofotômetro, no comprimento de onda de $535 \mathrm{~nm}$ para antocianinas e $374 \mathrm{~nm}$ para flavonoides. Os teores de antocianinas e flavonoides foram calculados por meio da fórmula: absorbância $x$ fator de diluição/98,2, e os resultados foram expressos em mg.100-1.

\section{Quantificação de pectina total}

As substancias pécticas totais foram extraídas e quantificadas segundo metodologia desenvolvida por McReady e MacComb (1952). Pesou-se $5 \mathrm{~g}$ de polpa em Becker, adicionando-se logo em seguida 25 $\mathrm{mL}$ de $\mathrm{EtOH}(95 \%)$, a amostra foi homogeneizada e deixada em repouso por um período de 30 minutos em geladeira. Após o repouso a amostra foi filtrada em um kitassato de $100 \mathrm{~mL}$, acoplado a uma bomba de vácuo, o resíduo foi lavado duas vezes com etanol a $75 \%$. Em seguida, o resíduo foi transferido para um becker de $50 \mathrm{~mL}$, onde se adicionou $40 \mathrm{~mL}$ de água destilada, homogeneizando novamente a amostra. Logo após, ajustou-se o pH da amostra para 11,5 com solução de $\mathrm{NaOH} 1 \mathrm{~N}$ para posterior repouso por 30 minutos, novamente em geladeira. A seguir o pH foi ajustado para 5,5 com ácido acético glacial diluído (5 mL de $\mathrm{CH} 3 \mathrm{COOH} / 50 \mathrm{~mL}$ de água destilada), para propiciar condições ideais de reação na amostra, após a inoculação de pectinase de Aspergillus niger v. Tiegem. Após a extração, foram realizadas as leituras quaduplicata, por espectrofotometria, a 520 $\mathrm{nm}$. A porcentagem de pectina total foi calculada por meio do peso da amostra, da absorbância obtida e da equação resultante da curva de calibração ( $x$ $=y-0,0064 / 0,00421)$, onde $0 y$ da equação foi substituído pela absorbância da amostra, obtendo-se o teor de pectina em micrograma $(\mu \mathrm{g})$, em seguida foi transformado em porcentagem por meio de uma regra de três simples.

\section{Quantificação de pectina solúvel}

As substancias pécticas solúveis foram extraídas e quantificadas segundo metodologia desenvolvida por McReady e MacComb (1952). Pesou-se $5 \mathrm{~g}$ de polpa em Becker, adicionando-se logo em seguida $25 \mathrm{~mL}$ de EtOH (95\%), a amostra foi homogeneizada e deixada em repouso por um período de 30 minutos em geladeira. Após o repouso a amostra foi filtrada em um kitassato de $100 \mathrm{~mL}$, acoplado a uma bomba de vácuo, o resíduo foi lavado duas vezes com etanol a $75 \%$. Em seguida o resíduo foi transferido para um erlenmeyer de $100 \mathrm{~mL}$, adicionando-se $40 \mathrm{~mL}$ de água destilada, a amostra foi agitada por 1 hora. Logo após, 0 material foi filtrado a vácuo e o sobrenadante diluído para $100 \mathrm{~mL}$ em balão volumétrico. Após a extração foram realizadas as leituras feitas em quaduplicata, por espectrofotometria, a $520 \mathrm{~nm}$. Para a quantificação da pectina solúvel foi utilizado o mesmo procedimento adotado para pectina total, ou seja, a porcentagem de pectina total foi calculada por meio do peso da amostra, da absorbância obtida e da equação resultante da curva de calibração $(x=y-0,0064 / 0,00421)$, onde $0 y$ da equação foi substituído pela absorbância da amostra, obtendo-se o teor de pectina em micrograma $(\mu \mathrm{g})$, em seguida foi transformado em porcentagem por meio de uma regra de três simples.

\section{Análise estatística}

Foi realizada uma análise estatística descritiva, onde foram obtidos os valores médios e o desvio padrão para cada parâmetro avaliado.

\section{RESULTADOS E DISCUSSÃO}

Os teores de antocianinas totais variaram de $1,51 \pm 0,24 \mathrm{mg} \cdot 100 \mathrm{~g}^{-1}$ a $1,66 \pm 0,47 \mathrm{mg}^{1} 100 \mathrm{~g}^{-1}$ para os frutos de cajá e umbu (Figura 1), respectivamente. As antocianinas são pigmentos presentes em vegetais, responsáveis pela coloração que variam do vermelho intenso ao violeta e azul, apresentando diversas funções para as plantas, atuando como antioxidantes, proteção contra os raios ultravioleta, mecanismo de defesa e função biológica. Além disso, quando presentes em alimentos atuam na prevenção do organismo contra ação dos radicais livres, causadores de inúmeras doenças (Formiga et al., 2016; Ribeiro et al., 2011). 
Figura 1. Teores médios de antocianinas totais em frutos de umbu (Spondias tuberosa Arr. Câm.) e cajá (Spondias mombin L.) procedentes da vegetação nativa de Alagoas.

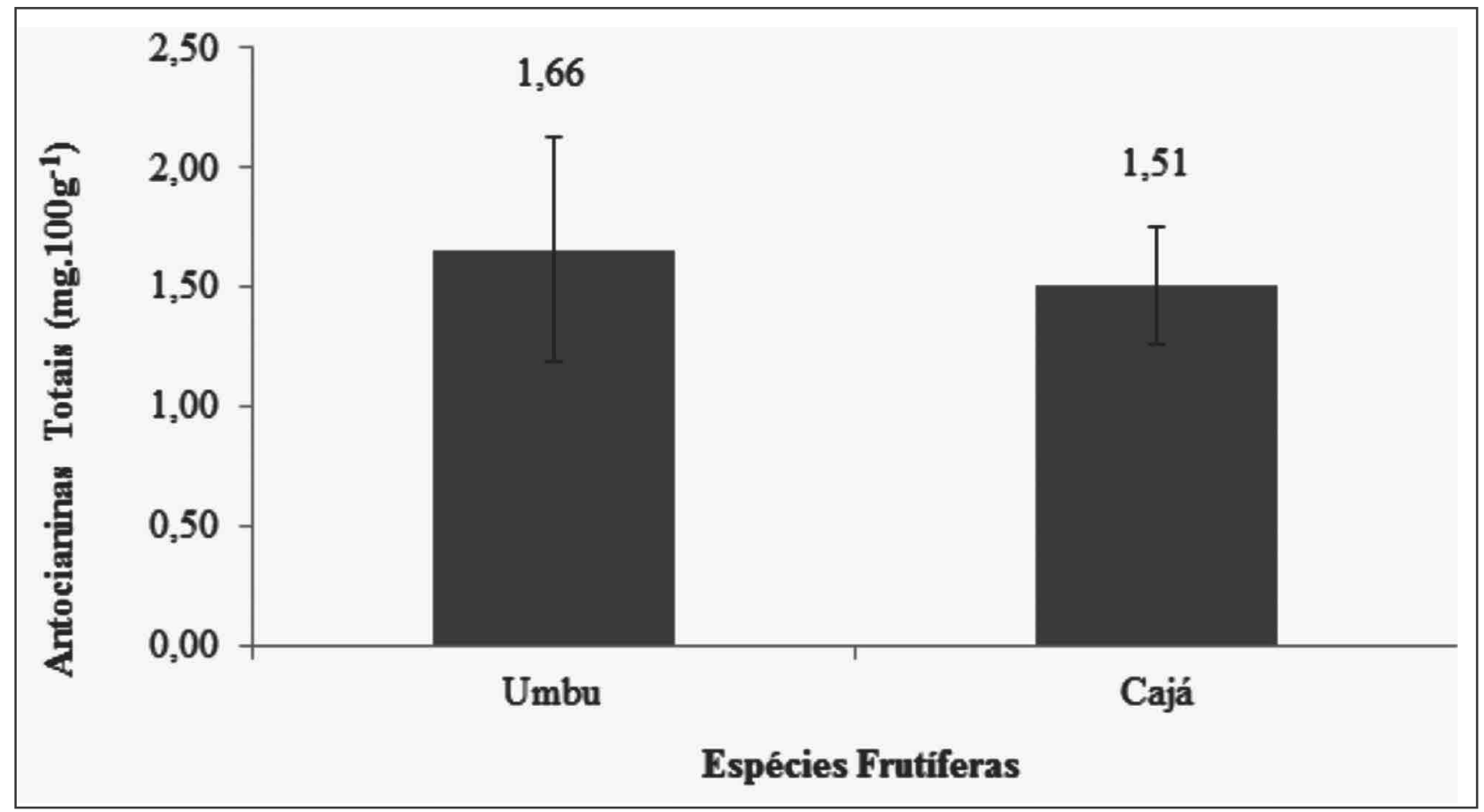

Em estudo realizado para quantificar os teores de compostos fenólicos em néctar de cajá enriquecido com farinha e extrato funcional, foi verificado valores de antocianinas totais variando de $0,07 \mathrm{mg}^{1} 100 \mathrm{~g}^{-1}$ a $0,17 \mathrm{mg} \cdot 100 \mathrm{~g}^{-1}$, teores inferiores aos constatados neste estudo (Souza et al., 2014). Almeida (2009) quantificou o teor de antocianinas totais em frutos de 14 genótipos de pedúnculos de Anacardium spp., verificando baixa quantidade deste pigmento nos genótipos avaliados, com concentrações variando de $0,88 \mathrm{mg} \cdot 100 \mathrm{~g}^{-1}$ a $0,97 \mathrm{mg} \cdot 100 \mathrm{~g}^{-1}$.

Em outra pesquisa realizada por Rocha et al. (2013), verificou-se concentração de antocianinas totais de $0,22 \mathrm{mg} 100 \mathrm{~g}^{-1} \mathrm{em}$ frutos de cajuí (Anacardium humile St. Hil.) nativos do cerrado piauiense. Observase que os frutos de $S$. mombin L. e S. tuberosa Arr. Câm. apresentaram conteúdo de antocianinas totais superiores aos verificados nestes estudos.

A concentração de flavonoides totais foi de $9,50 \pm 0,71 \mathrm{mg}^{1} 100 \mathrm{~g}^{-1}$ para os frutos de S. mombin L., enquanto que para os frutos de $S$. tuberosa Arr. Câm. foi verificado teor de $1,86 \pm 0,46 \mathrm{mg} \cdot 100^{-1}$. Vários fatores influenciam no teor de compostos fenólicos (antocianinas e flavonoides) em frutos, tais como: grau de maturação, espécie, técnicas de cultivo, origem geográfica, estágio de crescimento, condições de colheita e processo de armazenamento dos frutos, o que justifica as diferenças observadas na concentração destes compostos nos frutos analisados (Palioto et al., 2015).

Os flavonoides são compostos polifenólicos de origem natural, cuja síntese não ocorre na espécie humana. Estes fitoquímicos participam de importantes funções no crescimento, desenvolvimento, e na defesa dos vegetais contra 0 ataque de patógenos. Estando presentes em quase todas as plantas, concentrados em sementes, frutos, cascas, raízes, folhas e flores. Eles também possuem ação antioxidante, atuando sobre sistemas biológicos na estabilização de radicais livres, ajudando na prevenção de inúmeras doenças crônicas não transmissíveis (Dornas et al., 2007).

Zielinski et al. (2014), avaliaram os teores de compostos bioativos em 19 espécies frutíferas nativas do Brasil, e verificou concentração de flavonoides totais de $69,30 \mathrm{mg}^{1} 1000 \mathrm{~g}^{-1}$ e $87,13 \mathrm{mg}^{1} 1000 \mathrm{~g}^{-1}$ para os frutos de umbu e cajá, respectivamente. Silva et al. (2012) relataram valores de flavonoides amarelos em genótipos de Spondias mombin L. de pé-franco variando de $1,37 \mathrm{mg}^{1} 100 \mathrm{~g}^{-1}$ a $5,25 \mathrm{mg} \cdot 100^{-1}$. Nota-se que a concentração de flavonoides encontradas neste estudo para as mesmas espécies frutíferas nativas foram superiores. 
Figura 2. Teores médios de flavonoides totais em frutos de umbu (Spondias tuberosa Arr. Câm.) e cajá (Spondias mombin L.) procedentes da vegetação nativa de Alagoas.

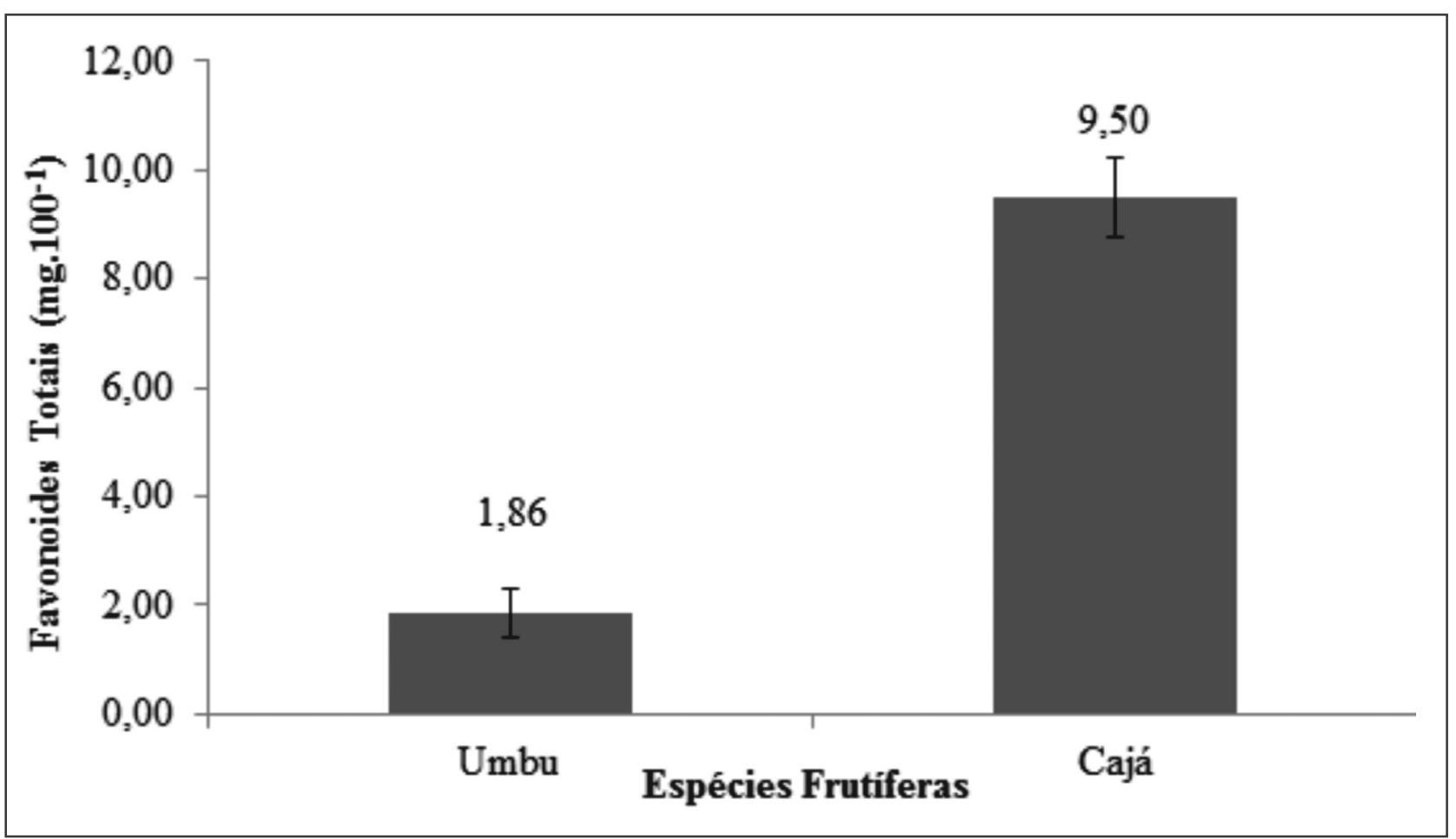

Nas Figuras 3 e 4 estão apresentados os total e solúvel em frutos de umbu e cajá praticamente valores médios de pectina solúvel e pectina total inexistem na literatura, sendo parâmetros importantes encontrados nos frutos de $S$. tuberosa Arr. Câm e $S$. mombin L. nativos de Alagoas. No fruto de umbu o teor de pectina solúvel foi $28,64 \pm 2,47 \%$ e de pectina total de qualidade, pois contribuem para a aceitação dos frutos do mercado consumidor, tanto para o consumo in natura como para o processamento. de $26,27 \pm 4,35 \%$. Informações sobre 0 teor de pectina

Figura 3. Teores médios de pectina solúvel em frutos de umbu (Spondias tuberosa Arr. Câm.) e cajá (Spondias mombin L.) procedentes da vegetação nativa de Alagoas.

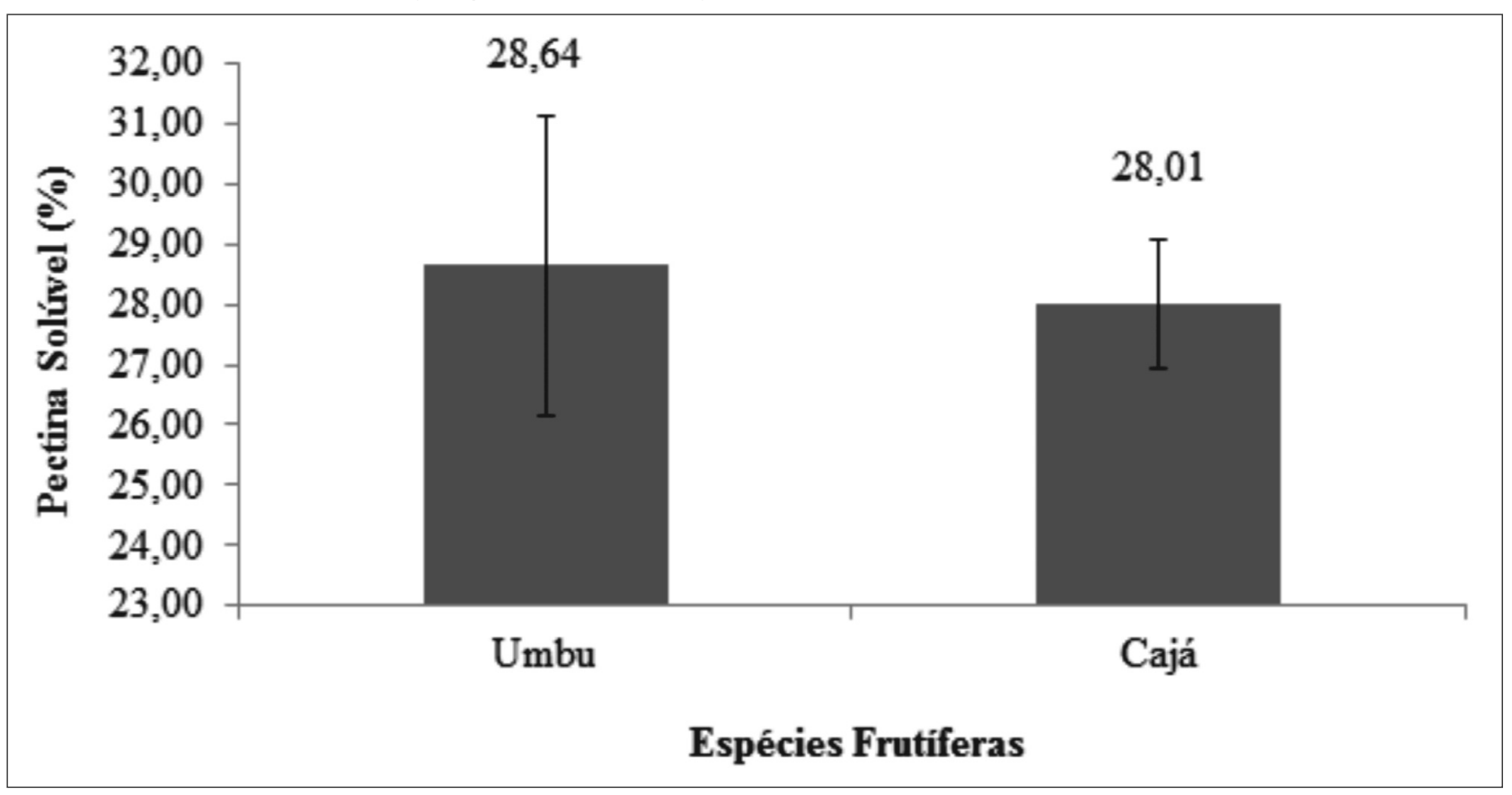


Figura 4. Teores médios de pectina total em frutos de umbu (Spondias tuberosa Arr. Câm.) e cajá (Spondias mombin L.) procedentes da vegetação nativa de Alagoas.

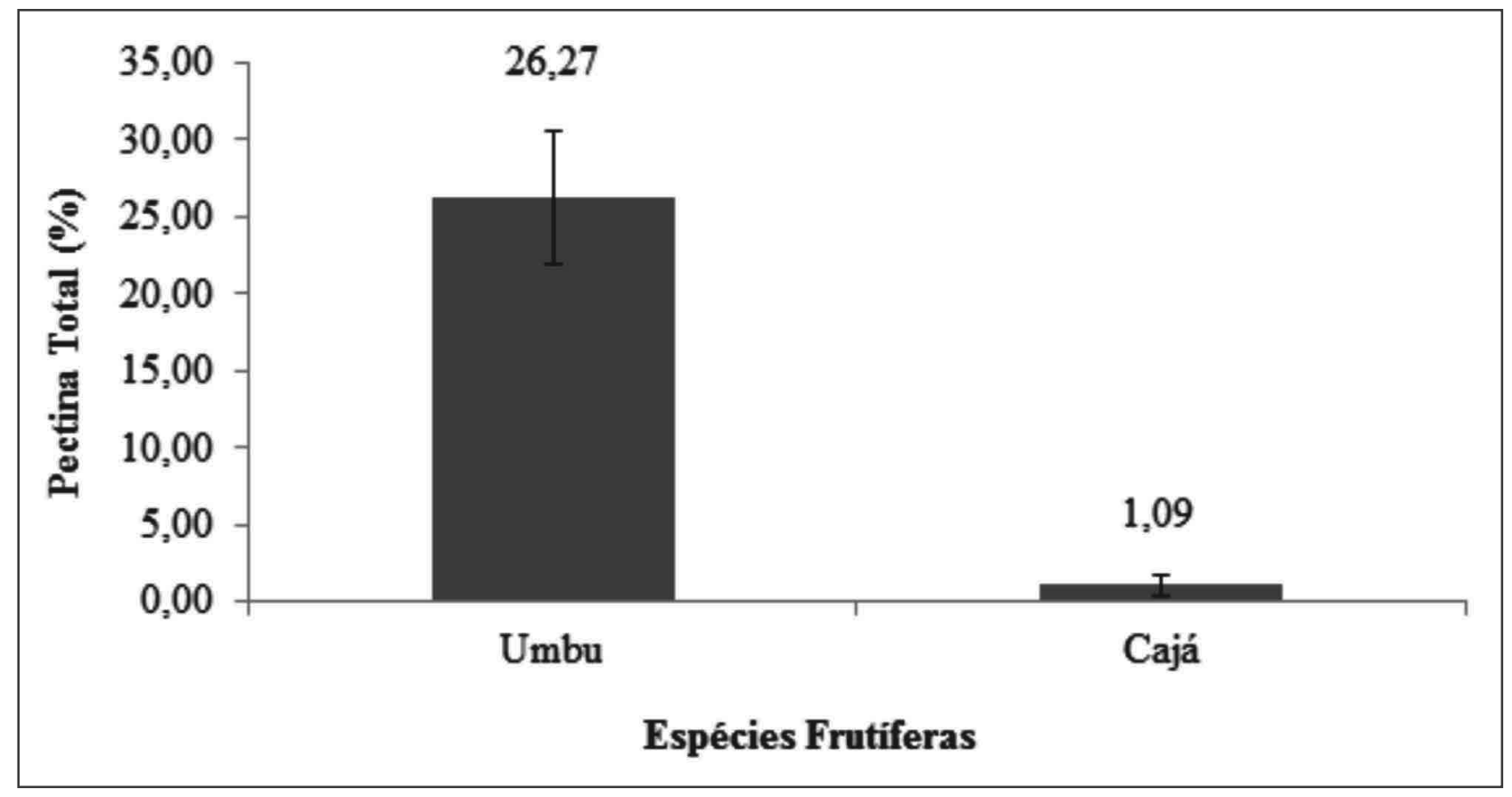

No trabalho realizado por Dias et al. (2007), onde foi realizado a caracterização física e físicoquímica de frutos de umbu, verificou que o teor de pectina total foi de $0,38 \%$. Silva et al. (2015), realizaram a caracterização físico-química de frutos dos genótipos de umbu-cajazeira (Spondias sp.) oriundos de IguatuCE, e observou valores variando de $0,15 \%$ a $0,64 \%$. Resultado superior foi reportado por Filho et al. (2012), que ao avaliarem as características físico-químicas da polpa de umbu encontrou $1,10 \%$ de pectina total.

Desta forma, como pode ser observado nos estudos citados acima, o teor destes compostos foi bastante variável. Segundo Chitarra e Chitarra (2005), o teor de compostos com propriedades biologicamente ativas são influenciados por diversos fatores, dentre eles, o genótipo, estádio de maturação, nutrição mineral e condições edafoclimáticas. Sendo assim, estes fatores explicam as diferenças observadas entre os teores destes compostos obtidos neste estudo, com as informações existentes na literatura para as mesmas espécies de ocorrência em diferentes regiões brasileiras, em virtude das características genotípicas potencias se expressarem em condições especificas de clima e solo de cada localidade geográfica.

Com relação ao fruto de cajá foi observado teor médio de $28,01 \pm 1,07 \%$ para pectina solúvel e $1,09 \pm 0,62 \%$ para pectina total. Figueiras et al.
(2000) encontraram teores inferiores ao realizar a caracterização físico-química de frutos de cajá, com teores médios de pectina solúvel variando $0,07 \%$ a $0,09 \%$ e pectina total de $0,28 \%$ a $0,13 \%$, para os frutos apresentando coloração predominantemente amarelo e amarelo, respectivamente. Rodrigues (2009) determinou o teor de pectina total em diversas frutas nativas, observando teor de 1,9\% para frutos $\mathrm{S}$. mombin L., ou seja, valor superior ao encontrado neste estudo.

As pectinas constituem um grupo de polissacarídeos estruturais presentes na parede celular primária e nas camadas intercelulares dos vegetais, contribuindo para a adesão entre as células e por conferir resistência mecânica a parede celular dos vegetais. Elas também apresentam um importante papel para processamento dos frutos, devido sua capacidade geleificante, texturizante, emulsificante e estabilizadora. Como também possuem importantes propriedades benéficas para a sua humana, pois atuam na estabilização da pressão sanguínea, auxilia em problemas gastrointestinais, atua favoravelmente nos níveis de colesterol sanguíneo, entre outras funções (Rodrigues, 2009).

Os frutos de S. tuberosa Arr. Câm. e S mombin L. analisados apresentam em sua composição substancial conteúdo de substâncias com propriedades funcionais, antocianinas e flavonoides, constituindo- 
se em uma fonte potencial de antioxidantes naturais para a dieta humana, auxiliando na prevenção de inúmeras doenças. Os frutos também apresentaram bons teores de pectina total e solúvel, indicando seu potencial tanto para o consumo in natura quanto para o processamento.

\section{AGRADECIMENTOS}

Os autores agradecem ao CNPq, A Fundação de Amparo à Pesquisa do Estado de Alagoas (FAPEAL) pelo apoio financeiro. Aos Laboratórios de Biotecnologia Vegetal e Fisiologia Vegetal do Centro de Ciências Agrárias da Universidade Federal de Alagoas pelo suporte físico e científico necessários para a realização desta pesquisa.

\section{REFERÊNCIAS BIBLIOGRÁFICAS}

Almeida, A. S. Qualidade, compostos bioativos, atividade antioxidante total de pedúnculos de cajueiros e frutos de umbuzeiro nativos do semiárido do Piauí. Tese (Dourado em Fitotecnia) - Universidade Federal Rural do Semiárido, Mossoró, 189 p., 2009.

Canuto, G.A.B; Xavier, A.A.O; Neves, L.C.; Benassi, M.T. Caracterização físico-química de polpas de frutos da Amazônia e sua correlação com a atividade antirradical livre. Revista Brasileira de Fruticultura, 2010, 32, 1196-1205.

Chitarra, M.I.F.; Chitarra, A. B. Pós-colheita de frutos e hortaliças: fisiologia e manuseio. Lavras: UFLA, 785 p., 2005.

Dias, S.L.; Dantas, J.P.; Araújo, J.P.; Barbosa, A.S.; Cavalcante, M.D,B.; Canuto, T.M.; Barbosa, A.S.; Rocha, C.O. Avaliação das características físicas e físico-químicas dos frutos de umbuzeiro. In: Congresso da Associação Norte-Nordeste de Química, 1., 2007, Natal. Resumos... Natal: Associação Norte-Nordeste de Química, 2007.

Dornas, W.C.; Oliveira, T.T.; Rodrigues-das-Dores, R.G.; Santos, A.F.; Nagem, T.J. Flavonóides: potencial terapêutico no estresse oxidativo. Revista Ciências Farmacêuticas Básicas Aplicadas, 2007, 28, 3, 241249.
Formiga, A.S.; Costa, F.B.; Silva, M.S.; Perreira, E.M.; Brasil, Y.L. Physical and chemical aspects of fruit Quipá (Tacinga inamoena). Revista Verde, 2016, 11, 5, 25-29.

Filgueiras, H.A.C.; Moura, C.F.H. Caracterização de frutas da América Latina. 5. ed. Jaboticabal: Funep, p. 66, 2000.

Francis, F.J. Analysis of anthocyanins. In: Markakis, P. (ed.). Anthocyanins as food colors. New York: Academic Press, 1982. p.181-207.

Lima, M.S.S.; Dantas, A.C.V.L.; Fonseca, A.A.O.; Barroso, J.P. Caracterização de frutos de genótipos selecionados de umbu-cajazeira (Spondias sp.). Interciência, 2015, 40, 5, 311-316.

McCread, P. M,;McComb, E.A.; Extraction end determination of total pectin materials. Analytical Chemistry, 1952, 24, 12, 1586-1588.

Menezes, P.H.S.; Souza, A.A.; Silva, E.S.; Medeiros, R.D.; Barbosa, N.C.; Soria, D.G. Influence of the maturation stage on the physical-chemical quality of fruits of umbu (Spondias tuberosa). Scientia Agropecuaria, 2017, 8, 73-78.

Oliveira, M.C.; Barcelos, N.P.; Curi, P.N.; Piuo, R.; Ferreira, D.F.N. Teores de macro e micronutrientes em frutas nativas do oeste do Mato Grosso do Sul. XXIV Congresso Brasileiro de Fruticultura, São Luís Maranhão, 2016.

Pimentel, B.M.V.; Francki, M.; Gollucke, B.P. Alimentos funcionais: introdução às principais substâncias bioativas em alimentos. São Paulo: Editora Varella, 2005.

Palioto, G.F.; Silva, C.F.G.; Mendes, M.P.; Almeida, V.V.; Rocha, C.L.M.S.C.; Tonin, L.T.D. Composição centesimal, compostos bioativos e atividade antioxidante de frutos de Morinda citrifolia Linn (noni) cultivados no Paraná. Revista Brasileira de Plantas Medicinais, 2015, 17, 1, 59-66.

Ribeiro, L.O.; Mendes, M.F.; Pereira, C.S.S. Avaliação da Composição Centesimal, Mineral e Teor de Antocianinas da Polpa de Juçaí (Euterpe edulis Martius). Revista Eletrônica TECCEN, 2011, 4, 2, 5-16. 
Rodrigues, L.M. Contribuição ao estudo bioquímico de frutas tropicais e exóticas produzidas no Brasil: pectina, açúcar e proteína. Dissertação (Mestrado) Universidade Estadual Paulista, 110 p., 2009.

Rocha, M.S.; Figueiredo, R.W.; Araújo, M.A.M.; Moreira-Araújo, R.S.R. caracterização físico-química e atividade antioxidante (in vitro) de frutos do cerrado piauiense. Revista Brasileira de Fruticultura, 2013, 35, 4, 933-941.

SEMARH-AL. Município de Maceió. Boletim Dezembro - 2015. Secretaria de Estado do Meio Ambiente e dos Recursos Hídricos. Maceió, AL. Janeiro, 2015.

Souza, D.G.; Santana, N.D.C.; Gomes, J.S.; Santos, A.F.; Vieira, M.M.S. Quantificação de compostos bioativos em néctar de cajá enriquecidos com farinha e extrato funcional. Caderno Verde de Agroecologia e Desenvolvimento Sustentável, 2014, 4, 1.

Santos, E.D., Lemos, E.E.P., Salvador, T. L.; Araújo, R. R., Barros, P.G., Rezende, L. P. Physico-Chemical Characteristics of Eight Genotypes of Cambuí (Myrciaria floribunda O. Berg) in Alagoas-Brazil In: 28th International Horticultural Congress, 2010, Lisboa. Book of Abstracts. Lisboa: International Society for Horticultural Science ISHS, 2010, 2, 175 - 175.

Silva, F.V.G.; Silva, S.M.; Silva, G.C.; Mendonça, R.M.N.; Alves, R.E.; Dantas, A.L. Bioactive compounds and antioxidant activity in fruits of clone and ungrafted genotypes of yellow mombin tree. Revista Ciência e Tecnologia de Alimentos, 2012, 32, 4, 685-691.
Souza, F.X.; Araújo, C.A.T. Avaliação dos métodos de propagação de algumas Spondias agroindustriais. Embrapa Agroindústria Tropical - Comunicado Técnico, 1999, 31, 4 p.

Souza, F.X. Spondias agroindustriais e os seus métodos de propagação. Fortaleza: Embrapa-CNPAT / SEBRAE/CE, 27 p., 1998.

Silva, F.V.G.; Silva, S.M.; Silva, G.C.; Mendonça, R.M.N.; Alves, R.E.; Dantas, A.L. Bioactive compounds and antioxidant activity in fruits of clone and ungrafted genotypes of yellow mombin tree. Ciência e Tecnologia de Alimentos, 2012, 32, 685-691.

Silva, L.R.; Alves, R.E.; Silva, S.M.; Nogueira, D.H. Caracterização físico-química de frutos dos genótipos de umbu-cajazeiras oriundos da microrregião de IguatuCE. Revista Brasileira de Tecnologia Agroindustrial, 2015, 9, 1, 1647-1659.

Teixeira, L.M.L.; Lemos, E.E.P.; Salvador, T.L.; Salvador, T.L.; Santos, H.R.S. Desempenho agronômico de cinco genótipos de cajá (Spondias mombin L.) sob condições de manejo agrícola. XXIV Congresso Brasileiro de Fruticultura, São Luís - Maranhão, 2016.

Zielinski, A.A.F.; Ávila, S.; Ito, V.; Nogueira, A.; Wosiacki, G.; Haminiuk, C.W.I. The Association between Chromaticity, Phenolics, Carotenoids, and In Vitro Antioxidant Activity of Frozen Fruit Pulp in Brazil: An Application of Chemometrics. Journal of Food Science, 2014, 79, 4, 510-516. 